Revue européenne des sciences sociales

European Journal of Social Sciences

XXXVIII-117| 2000

Métaphores et analogies. Schèmes argumentatifs des sciences sociales

\title{
De quelques analogies physiques en économie politique
}

\section{Claude Mouchot}

\section{CpenEdition}

Journals

Édition électronique

URL : http://journals.openedition.org/ress/715

DOI : $10.4000 /$ ress. 715

ISSN : 1663-4446

Éditeur

Librairie Droz

Édition imprimée

Date de publication : 1 février 2000

Pagination : 121-130

ISBN : 2-600-00409-2

ISSN : 0048-8046

Référence électronique

Claude Mouchot, « De quelques analogies physiques en économie politique », Revue européenne des sciences sociales [En ligne], XXXVIII-117 | 2000, mis en ligne le 17 décembre 2009, consulté le 01 mai 2019. URL : http://journals.openedition.org/ress/715; DOI : 10.4000/ress.715

(c) Librairie Droz 


\section{Claude MOUCHOT}

\section{DE QUELQUES ANALOGIES PHYSIQUES EN ÉCONOMIE POLITIQUE}

Il est bien connu que les fondateurs de la théorie dite néoclassique, Walras et Pareto (mais aussi bien d'autres...), ont abondamment utilisé les analogies qu'il pensaient découvrir entre les «équilibres » qu'ils élaboraient et divers équilibres définis en physique et notamment en mécanique.

Nous disons «les équilibres », car, en amont de l'analogie appliquée à l'équilibre général (qui nous paraît surtout incantatoire), il y a eu des tentatives de construction d'analogies beaucoup plus concrètes appliquées à l'équilibre du consommateur.

Ce sont celles-ci que nous présenterons en premier lieu.

Nous montrerons ensuite que, bien avant les néoclassiques, Quesnay et les Classiques ont élaboré des équilibres, eux aussi «analogues» à des équilibres physiques, même si le mot équilibre et la référence à la physique sont absents de leurs écrits. Nous montrerons aussi que l'équilibre général, de ce point de vue, ne diffère des précédents que par l'explicitation consciente et volontaire de l'analogie. Il y a plus : les trois «équilibres » étudiés possèdent des caractéristiques communes qui, à notre sens, soulèvent une question fondamentale, celle du statut de notre discipline.

Nous proposerons donc une réponse à cette question en troisième partie.

\section{I. - L'ÉQUILIBRE DU CONSOMMATEUR}

\section{A. Analogies ou obsession?}

Rappelons d'abord que, pour Walras, «l'économie pure est une science physico-mathématique», en notant tout de suite que personne n'a jamais défini une science physico-mathématique! Mais logique avec lui-même, il n'a pas cessé de rechercher explicitement des analogies entre l'équilibre du consommateur et divers équilibres physiques. En voici quelques exemples.

$1^{\circ} \quad$ La métaphore de la masse

«Philosophiquement je pose:

$$
\begin{aligned}
& \mathrm{p}=\mathrm{g}+\mathrm{g}+\mathrm{g}+\ldots=\mathrm{mg} \\
& \mathrm{p}^{\prime}=\mathrm{g}+\mathrm{g}+\mathrm{g}+\mathrm{g}+\ldots=\mathrm{m}^{\prime} \mathrm{g} \quad \text { soit } \quad \mathrm{p}^{\prime} / \mathrm{p}=\mathrm{m}^{\prime} / \mathrm{m}
\end{aligned}
$$

c'est-à-dire que j'appelle masse ou $\mathrm{m}$ une cause proportionnelle au poids.

Et de même je pose:

c'est-à-dire que j'appelle rareté ou $\mathrm{r}$ une cause proportionnelle à la valeur d'échange» (Euvres économiques complètes, vol. XI, p. 629). 
$2^{\circ}$ La métaphore de la force vive

$\ll \mathrm{V}_{\mathrm{a}} / \mathrm{V}_{\mathrm{b}}=\mathrm{r}_{\mathrm{a}} / \mathrm{r}_{\mathrm{b}} \quad$ Donc, la satisfaction maxima a lieu par la proportionnalité des raretés aux valeurs $[\ldots]$ vitesse...

...si on prend pour mesure de la force, la force vive, c'est-à-dire la force multipliée par sa

$\mathrm{P} / \mathrm{Q}=\mathrm{p} / \mathrm{q} \quad \mathrm{C}$ 'est-à-dire que l'équilibre de la romaine a lieu par la proportionnalité des forces aux bras de levier.

L'analogie est évidente. Aussi a-t-on déjà signalé celle des forces et des raretés...» (Euvres économiques complètes, vol. VII, pp. 333-334).

La dernière phrase nous conduit à:

$3^{\circ}$ La métaphore de la force

$\begin{array}{ll}\text { «En mécanique } & \text { En économique } \\ \text { Une molécule } & \text { Un individu } \\ \ldots & \ldots \\ \text { Force } & \text { Utilité ou désutilité limite } \\ \ldots & \ldots \\ & (\text { Fisher, p. } 85) »\end{array}$

(Euvres économiques complètes, vol. XI, p. 623).

Si on tient compte du fait que, dans le $3^{\circ}$, Walras n'a fait que recopier Fisher et que donc il faut remplacer utilité limite par rareté, on se trouve en présence de trois analogies: masse, force et force vive; choisissez celle qui vous convient le mieux!

Il est clair qu'il n'est même plus question d'analogies, mais seulement de ressemblances formelles qui ne pourraient prendre de l'intérêt qu'à partir du moment où de véritables correspondances entre les termes seraient mises en évidence.

Eh bien, c'est précisément ce qui se passe pour le $3^{\circ}$.

\section{B. Analogie forcée - impasse assurée:}

\section{le consommateur et la théorie des champs}

$1^{\circ}$ L'analogie entre

I. le comportement du consommateur soumis au «champ de forces » que constitue sa recherche du maximum d'utilité et à sa contrainte budgétaire d'une part, et

II. Le comportement d'un point matériel dans un «bol» soumis au champ de forces de la pesanteur et à la contrainte de rester sur la paroi du bol d'autre part est explicite maintenant dans tous les manuels. On peut l'illustrer par les deux schémas suivants.

Le premier à avoir utilisé cette analogie semble bien être I. Fisher en 1892 et Walras n'a fait que reprendre les correspondances que Fisher avait proposées (nous en avons donné quelques-unes ci-dessus). Elle semble parfaite:

- le consommateur (fig. 2) se trouvant en A désire se déplacer dans le sens de F; la contrainte budgétaire l'oblige à demeurer sur la droite $\mathrm{AB}$ et il atteint le point $\mathrm{B}$, où il obtient le maximum de satisfaction; 
- le point matériel (fig. 1) se trouvant en A désire se déplacer dans le sens de F; la paroi du bol l'oblige à demeurer sur elle et il atteint le point $\mathrm{B}$, où son énergie potentielle est minimale. On notera que la «colline des plaisirs » est exactement une portion de bol renversé...

Analogie parfaite donc, mais en termes de vecteurs, d'êtres mathématiques. Or pas plus Walras que Fisher ou Pareto (ou certains auteurs actuels) n'ont fait la différence entre un vecteur (être mathématique) et une force, être physique (ce qui montre au passage le non-sens d'une science physico-mathématique). Pareto en particulier utilise souvent des expressions du type «la force de son désir le pousse à...».

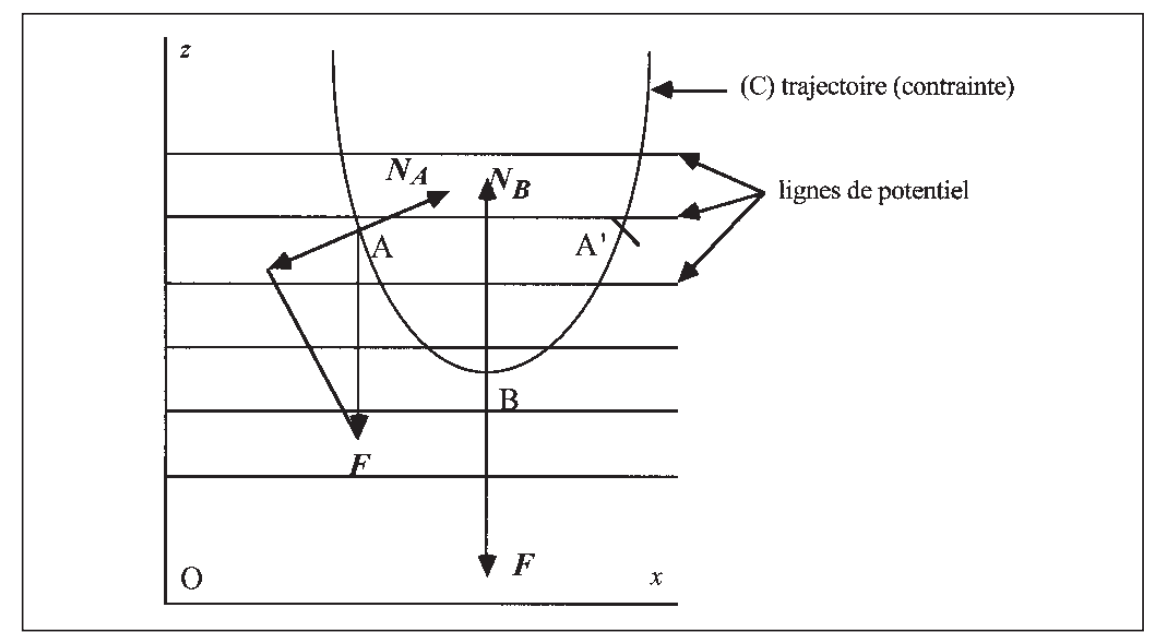

Figure 1

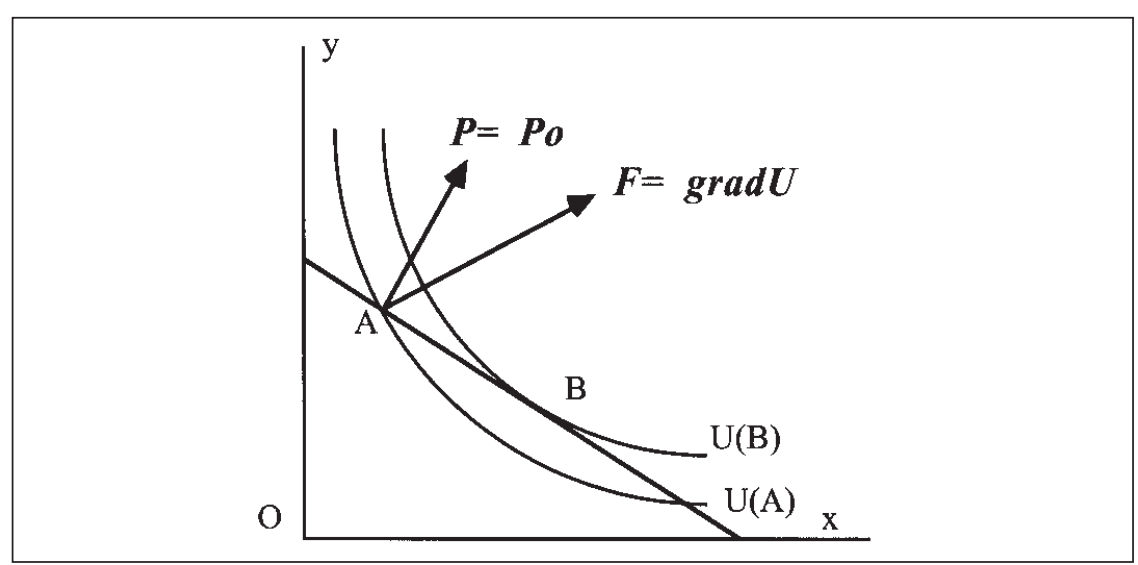

Figure 2 
Tous ces auteurs se sont donc engagés sur la voie de l'analogie avec un champ de forces physiques, alors que cette analogie est fausse. Et elle est fausse pour une raison très simple, c'est qu'il n'y a aucune force physique qui s'exerce sur le point A de la figure 2. Une force ne peut s'exercer que sur un corps, réduit à un point matériel en mécanique pure, et surtout doté d'une masse. Or le point A ne possède aucune de ces caractéristiques; il n'existe que dans l'esprit du consommateur. Si d'ailleurs, la notion de force physique pouvait s'appliquer, c'est que A aurait une masse; mais alors le consommateur n'atteindrait jamais le point $\mathrm{B}$ : comme en mécanique pure, il oscillerait indéfiniment autour de B sans jamais y rester!

Il est pourtant un point de l'analogie qui aurait pu inquiéter Fisher. Il prend «l'individu» (le consommateur) comme analogue de «la molécule» (le point matériel). Cela n'a aucun sens : l'individu, en tant que tel, ne se trouve pas au point A de la figure 2; tout au plus peut-on y voir le panier de biens X et Y dont il dispose au départ. Mais ce panier, lui, n'est tiré ou poussé par aucune force...

Jusque-là, rien de bien grave: on utilise improprement le mot «force» mais l'analogie reste valable en termes de «vecteurs».

Hélas, il y a effectivement quelque chose de beaucoup plus grave. C'est que, partis sur l'idée de force et d'équilibre statique, ces auteurs ont tous cru qu'ils allaient pouvoir développer une dynamique, exactement comme l'avait fait la physique.

Puisque le vecteur gradU est assimilé (faussement) à une force, il doit pouvoir expliquer le repos et le mouvement. Il suffit de rajouter une nouvelle analogie, purement formelle cette fois, entre les équations différentielles:

$$
r_{a} d q_{a}+r_{a} d q_{a}=0 \quad \text { et } \quad \mathrm{P} d p+\mathrm{Q} d q=0 \quad \text { (Walras) }
$$

pour qu'on se croie à l'orée d'une théorie dynamique.

Il semble bien que ce soit Pareto qui ait placé le plus d'espoir dans cette direction. Il voulait décrire les phénomènes de marché par un système d'équations différentielles (susceptibles en physique de rendre compte à la fois du repos et $\mathrm{du}$ mouvement) et c'est exactement l'impasse annoncée. De plus, le fondement de cette impasse est bien celui que nous indiquons puisqu'il considérait le marché comme un système de points matériels mus par des forces, faisant ainsi la même erreur que Fisher.

Il montre souvent la déception de ne pas voir cet espoir se réaliser; il ne pouvait qu'en être ainsi puisque les bases d'une dynamique ne sont pas présentes dans l'analogie considérée.

Mais, comme nous allons le montrer, l'équilibre général n'est pas le seul, ni le premier, des équilibres élaborés par les théories économiques à se fonder sur des analogies physiques et à n'être que statique. Cela nous conduira à nous demander s'il peut en être autrement.

\section{II. - UNE ÉCONOMIE EN ÉQUILIBRE? QUESNAY, CLASSIQUES ET NÉOCLASSIQUES}

\section{A. Le Tableau économique et la métaphore du flux}

On fournit une présentation du Tableau, faite par Quesnay en 1766.

La métaphore du flux est évidente, mais elle appelle de nombreuses remarques. 
$1^{\circ}$ L'activité économique est ainsi présentée tout à la fois comme mouvement puisque les sommes circulent; mais aussi comme un mouvement qui ne mène nulle part puisque l'économie nationale se reproduit identique à elle-même d'année en année. C'est bien évidemment la définition de l'état stationnaire: le mouvement des parties n'empêche pas le tout de rester au repos. Équilibre donc, tout au moins d'un point de vue global.

$2^{\circ}$ L'analogie avec la circulation sanguine est elle aussi évidente et il ne faut pas oublier que Quesnay était médecin du Roy Louis XV et que la circulation sanguine est connue depuis 1616.

Sommes qui servent à payer le revenu et les intérêts des avances primitives

Dépenses des avances

\section{Formule du tableau économique Reproduction totale : 5 milliards}

\begin{tabular}{|c|c|c|}
\hline $\begin{array}{l}\text { AVANCES } \\
\text { annuelles } \\
\text { de la classe } \\
\text { productive }\end{array}$ & $\begin{array}{l}\text { REVENU } \\
\text { Pour les } \\
\text { propriétaires } \\
\text { des terres } \\
\text { et le souverain }\end{array}$ & $\begin{array}{l}\text { AVANCES } \\
\text { de la classe } \\
\text { stérile }\end{array}$ \\
\hline 2 milliards & 2 milliards & -1 milliard \\
\hline 1 milliard - & & 1 milliard \\
\hline 1 milliard & & - 1 milliard \\
\hline 1 milliard & & \\
\hline 2 milliards & & $\begin{array}{l}\text { Total2 milliards } \\
\text { dont la moitié } \\
\text { est retenue } \\
\text { par cette classe } \\
\text { pour les avances } \\
\text { de l'année suivante }\end{array}$ \\
\hline
\end{tabular}

Total

$3^{\circ}$ La question du «moteur » de cette circulation ne semble pas avoir été soulevée par Quesnay. Tout comme la circulation sanguine est expliquée par l'énergie du cœur, il explique la circulation économique par la «production» de la terre (d'où le nom de «classe productive» donné aux agriculteurs); on notera que, pour nous, le Tableau ne fonctionne, les flux n'existent, que par le travail des deux classes, productive et stérile. Et le fait que Smith et surtout Ricardo élaboreront une théorie de la valeur travail provient sans doute de la connaissance qu'a eue Smith du Tableau.

$4^{\circ}$ On peut aussi constater que ce schéma correspond à ce que les anthropologues ont appelé les «sociétés traditionnelles » (sous réserve de définir autrement les classes sociales): sociétés sans histoire, puisque se reproduisant (presque) à l'identique. Nous retrouverons cette vacuité du temps en B et en C. 
Mais le plus important peut-être, c'est de constater que la première représentation globale de l'économie, fondée sur l'analogie d'un flux biologique, est représentation d'un état stationnaire, d'un «équilibre» globalement statique, qui ne correspond pas à la réalité économique puisque celle-ci, même au niveau global, est constamment en mouvement.

Notons aussi que, à l'exception de Marx, il faudra attendre Keynes pour voir réapparaître une représentation du «circuit économique». Or, pour Marx et pour Keynes (selon des modalités évidemment différentes), il n'était pas question de se représenter un état stationnaire; et, ce qui est frappant, c'est que, pour chacun de ces deux auteurs, il y a presque autant d'interprétations de ce qu'ils ont écrit que de lecteurs. L'unanimité (de compréhension, d'interprétation) ne peut-elle se faire qu'autour, ou à partir, d'un «équilibre»?

\title{
B. Les Classiques: prix naturel et équilibre de long terme
}

\begin{abstract}
Dans chaque société... il y a un taux moyen ou ordinaire pour les salaires, pour les profits... et pour les fermages [...]. On peut les appeler taux naturels des salaires, des profits et des fermages [...]. Lorsque le prix d'une marchandise n'est ni plus ni moins que ce qu'il faut payer, suivant leurs taux naturels, les salaires, les profits et les fermages employés à produire cette marchandise, alors elle est vendue à ce qu'on peut appeler son prix naturel [...]. Le prix actuel d'une marchandise est ce qu'on appelle son prix de marché. Il peut être ou au-dessus, ou au-dessous, ou précisément au niveau du prix naturel [...].

Le prix naturel est donc pour ainsi dire le point central vers lequel gravitent continuellement les prix de toutes les marchandises [...].

Lorsque la quantité mise sur le marché suffit tout juste pou remplir la demande effective, le prix de marché se trouve naturellement, au moins autant qu'il est possible d'en juger, le même que le prix naturel.

Tant que cette demande reste la même, le prix de marché doit vraisemblablement aussi rester le même et être tout à fait le même que le prix naturel, ou du moins, aussi rapproché qu'il est possible d'en juger (A. Smith).
\end{abstract}

À nouveau, le terme d'équilibre est absent. Mais il se donne à voir avec le mot «gravitation», lequel renvoie bien à la métaphore physique. Cette gravitation sera d'ailleurs reprise par L. Walras (pour une tout autre définition du prix) et illustrée par l'image, elle aussi physique, des vagues sur un lac qui tendent à retrouver le niveau du lac au repos (il observait sans doute attentivement le lac Léman!).

On pourrait penser que, comme en physique, il s'agit de remplacer une multitude de mesures (de prix, de niveaux) par quelque chose comme une moyenne. Mais cette métaphore du lac (ou celle du pendule, ou toute autre analogie provenant de la physique) induit ici un élément étranger à la réalité économique. En effet, le niveau du lac au repos (la position du pendule au repos) existe et est observable (il est d'ailleurs une moyenne des niveaux des différentes parties du lac). Alors que le prix naturel n'a pas d'existence et n'est pas observable (et en tout état de cause il n'est pas la moyenne de tous les prix de marché); il n'est qu'un prix construit par le théoricien comme Smith le sait bien qui précise «autant qu' il est possible d'en juger».

Le prix naturel, le prix d'équilibre de la théorie économique «classique», n'aura donc absolument pas le même statut que le niveau d'équilibre de l'eau d'un lac. Répétons-le: celui-ci existe; celui-là ne peut venir à l'existence qu'à long terme, c'est-à-dire jamais. 
En effet, alors que parfois il n'y a pas de vent sur le lac et donc qu'il est alors au repos, la réalité économique, elle, n'est jamais au repos; elle crée constamment des déséquilibres qui sont autant d'obstacles à l'instauration du prix naturel. Il va donc falloir construire une économie «au repos ». Trois hypothèses y pourvoiront, qui ont chacune partie liée avec le temps:

- la mobilité parfaite des capitaux (qui permet un ajustement immédiat de l'offre à la demande);

- l'investissement immédiat de l'épargne (qui est un aspect de la loi de Say: toute offre crée sa propre demande);

- Les «fonctions de production» (anachronisme bien sûr) ne varient pas.

Ayant ainsi supprimé: les délais d'ajustement, le problème de la demande (loi de Say) et l'évolution technique des entreprises, il devient possible de conduire cette «économie» au repos par le seul jeu de la concurrence qui fera émerger, à long terme, les prix naturels. Il est clair, d'une part, que ces prix n'ont en réalité rien de naturel et, d'autre part, que le long terme en question est parfaitement fictif et théorique: c'est un temps conceptuel, sans contenu, puisqu'il ne s'y passe quasiment plus rien. La seule «activité » qui habite ce temps est l'équilibration des prix par la seule concurrence, exactement comme, lorsque le vent s'arrête, le niveau du lac devient homogène par la seule pesanteur - et nous retrouvons la métaphore.

On retrouve ainsi, dans ce long terme, un état stationnaire (il ne s'agit évidemment pas de l'état stationnaire que Smith comme Ricardo, chacun selon des voies différentes, croyaient pouvoir prédire comme aboutissement de l'évolution de l'économie réelle). Partie de fondements très différents de ceux de Quesnay, la théorie classique, s'appuyant implicitement sur des analogies physiques, ne parvient, elle aussi, à donner de la réalité économique, essentiellement mouvante, qu'une représentation statique.

De plus, le prix (!) à payer pour parvenir à cette représentation de l'économie paraît bien lourd puisqu'on a construit des prix qui n'apparaîtront jamais dans la réalité. On ne «représente» plus la réalité; on construit un objet de pensée dont quelques éléments sont bien des représentations de cette réalité, mais dont les autres éléments n'ont plus aucun rapport avec celle-ci.

Mais cela, les Classiques, et spécialement les Ricardiens (J.S. Mill, Cairnes, J. Neville Keynes...) le savaient qui parlaient de «vérités abstraites». Ils avaient conscience que les «vérités», les «lois», économiques qu'ils élaboraient n'étaient que partielles et demandaient à être replongées dans une appréhension plus vaste de la réalité sociale.

\section{Les néoclassiques et l'équilibre général}

À partir de Walras, apparaissent explicitement, et le mot équilibre, et l'analogie avec un équilibre physique. Rappelons ces phrases bien connues:

«L'équilibre économique est l'état qui se maintiendrait indéfiniment s'il n'y avait aucun changement dans les conditions dans lesquelles on l'observe...

Il est déterminé de telle sorte que, s'il n'est que faiblement modifié, il tend immédiatement à se rétablir... 
Il nous faudra exprimer mathématiquement que, l'état d'équilibre étant atteint..., le système se maintient indéfiniment dans l'état considéré» (Pareto).

Accessoirement on notera la force de persuasion de l'utilisation successive du conditionnel, du présent et enfin du futur pour que soit admise, sans autre démonstration, l'existence de l'équilibre.

Les phrases de Pareto sont parfaitement explicites: en les lisant, on «voit» le pendule, le lac, la bille dans un bol. Mais, pour ce qui nous concerne, elles ont un avantage: la troisième nous montre que cette nouvelle théorisation retrouve les précédentes au moins sur un point: nous sommes à nouveau en présence d'un état stationnaire. Et nous avons déjà signalé la déception de Pareto de ne pouvoir aller au-delà d'une représentation statique.

Rien d'étonnant à cela puisque l'atteinte de l'équilibre n'a de sens que dans un temps aussi vide ici qu'il l'était pour les Classiques - et cela est logique puisqu'ils font les mêmes hypothèses que précédemment en y ajoutant la non-variation des fonctions d'utilité. Mêmes causes, mêmes conséquences.

Il y a toutefois une différence essentielle entre eux.

On l'a déjà dit: les Classiques savaient qu'ils n'atteignaient que des «vérités abstraites». Il n'en est pas de même des néoclassiques qui croient eux qu'ils détiennent la Vérité de l'économie. Depuis les Chicago boys jusqu'aux tenants actuels de l'économie de l'offre, tous connaissent, d'une connaissance sûre, les lois de l'économie et les appliquent... pour le malheur de populations entières.

L'analogie, implicite mais qui n'était, et consciemment, que partielle, chez les Classiques, est devenue homologie chez les néoclassiques : leur modèle représente exactement (?), point par point (?), la réalité; il fournit donc (?) les solutions aux problèmes que pose cette réalité. Quand cette réalité semble quelque peu se rebiffer, ne pas entrer facilement dans le cadre, il existe un sésame: la réalité impure comporte des «frottements » que la théorie pure a négligés. Et nous revoilà dans l'homologie.

Il reste un petit problème: en physique, il a été possible d'élaborer des théories du frottement. En économie au contraire, à chaque fois qu'on désire intégrer un «frottement» dans la théorie, c'est l'équilibre général qu'on perd — et la plupart du temps pour cause de non-convexité de certaines fonctions. Cela n'empêche pas la théorie de continuer à se croire, et à se proclamer, la Vérité de l'économie.

Trois représentations de l'économie,

trois analogies, implicites ou explicites, avec les sciences «dures»,

trois états stationnaires...

Se pose alors la question suivante:

la représentation de l'activité économique comme état stationnaire est-elle une nécessité de notre esprit (l'analogie physique ne jouant alors qu'un rôle d'illustration) ou, au contraire, est-ce l'analogie qui impose à notre esprit de ne plus pouvoir se représenter cette activité que comme stationnaire? 


\section{III. - LE PROBLÈME EST EN AMONT DE L'ANALOGIE}

Dans les trois exemples ci-dessus, nous avons insisté sur le fait que le temps pris en compte par les théories correspondantes était un temps vide, contredisant ainsi la réalité du temps économique. Et, contrairement à ce qu'on pourrait penser après ce que nous avons exposé ci-dessus, nous ne pensons pas que ce soit les analogies physiques, en tant que telles, qui soient la cause de cette vacuité.

Pour le dire autrement, à la question posée à la fin du paragraphe précédent, nous répondrons, dans un premier temps, que ces analogies sont secondes par rapport à une des nécessités de notre esprit: le besoin de cohérence logique. Nous montrerons, dans un deuxième temps, que ce besoin de cohérence logique est luimême second par rapport à une autre nécessité de notre esprit: le besoin d'appréhension globale du monde dans lequel nous vivons et dans lequel nous voulons pouvoir inscrire notre action.

\section{A. Cohérence logique et vacuité du temps}

Toute théorie, si le mot a un sens, ne peut être que cohérente, logique.

Mais en même temps tout le monde a toujours su que le discours logique ne faisait que dérouler les conséquences des hypothèses sur lesquelles il est fondé.

Et tout est dit: lorsque l'individu est «rentré» dans les hypothèses d'une théorie, il ne peut plus être, dans cette théorie, qu'un « robot intelligent». C'est évident pour homo æeconomicus; ce l'est tout autant pour les capitalistes ou les rentiers des Classiques; ce l'est encore pour les membres des classes sociales de Quesnay. L'individu ne «vit» plus, il déroule comme une particule les conséquences nécessaires de ses «conditions initiales». L'analogie physique n'est alors bien qu'une illustration de ce déroulement des «conséquences passives», comme le dit Bachelard.

Et le temps de la physique classique n'est que le support, neutre, de ce déroulement; il est effectivement vide; il «coule».

Or, l'action humaine peut précisément être définie comme rupture, au moins partielle, de ces conséquences passives; comme surgissement du nouveau; elle donne un contenu au temps. Dans l'action, et c'est à nouveau Bachelard, «le temps ne coule plus, il jaillit».

Est-il alors possible de conserver « de la théorie » et, malgré cela, de donner du contenu au temps?

\section{B. Rationnel et raisonnable}

Oui, cela est possible et les Classiques l'ont fait. Ils avaient vu et ils affirmaient que les vérités abstraites de leur théorie devaient être nuancées, voire contredites, par d'autres logiques issues d'autres domaines. Ils savaient, et ils disaient, que la logique de leur objet (l'économie) était limitée par ces autres logiques.

Après avoir élaboré leur théorie par une démarche rationnelle (et qui dira que Ricardo manquait de logique?), ils ne craignaient pas de devenir raisonnables en abandonnant la rationalité pure pour prendre en compte des éléments de la réalité sociale que leur théorie ne pouvait pas logiquement intégrer.

Attitude raisonnable donc, mais qui ne s'oppose pas à rationnelle: elle est «autre». En effet, si cette prise en compte d'éléments étrangers ne peut pas, par 
hypothèse, être déduite, elle peut parfaitement être argumentée. Ce mot, à lui seul, réintroduit d'un seul coup le choix, le risque, la décision, le conflit des opinions... en un mot la réalité de la vie sociale et politique. Pour eux, l'économie était réellement politique.

Nos «économistes» actuels ne sont plus que rationalistes.

Quand redeviendront-ils raisonnables?

Faculté des sciences économiques

Université Lumière-Lyon 2 